From the University Hospital, Hamburg Eppendorf; South West German Comprehensive Cancer, University Hospital Tübingen, Tübingen; Merck Serono, Darmstadt Germany; State Medical Academy, City Clinical Hospital \#4, Dnepropetrovsk, Ukraine; Moscow City Oncology Hospital \#62, Moscow Area, Russian Federation; Hospital Universitario La Fe, Valencia, Spain; Istituto Europeo di Oncologia, Milan, Italy Institutul Oncologic "Prof. Dr. Al. Trestioreanu" Bucuresti, Bucharest, Romania; Wilhelminenspital der Stadt Wien, Vienna, Austria; and Wojewódzki Szpital Specjalistyczny Oddzia Chemioterapii, Krakow, Poland.

Submitted October 31, 2008; accepted November 25, 2008; published online ahead of print at www.jco.org on December 29, 2008

Presented at the 43rd Annual Meeting of the American Society of Clinical Oncology, June 1-5, 2007, Chicago, IL; 14th European Cancer Conference, September 23-27, 2007, Barcelona, Spain; 44th Annual Meeting of the American Society of Clinical Oncology, May 30-June 3, 2008, Chicago, IL; and The ESMO International Symposium: 10th World Congress on Gastrointestinal Cancer, June 25-28, 2008, BarceIona, Spain.

Authors' disclosures of potential conflicts of interest and author contributions are found at the end of this article.

Clinical Trials repository link available on JCO.org.

Corresponding author: Carsten

Bokemeyer, MD, Universitätsklinikum Hamburg-Eppendorf, Martinistrasse

52, 20246 Hamburg-Eppendorf, Germany; e-mail: c.bokemeyer@uke.uni-

hamburg.de.

The Appendix is included in the full-text version of this article, available online at www.jco.org. It is not included in the PDF version (via Adobe® Reader $\circledast$ )

(C) 2008 by American Society of Clinical Oncology

0732-183X/09/2705-663/\$20.00

DOI: $10.1200 / J C O .2008 .20 .8397$

\title{
Fluorouracil, Leucovorin, and Oxaliplatin With and Without Cetuximab in the First-Line Treatment of Metastatic Colorectal Cancer
}

Carsten Bokemeyer, Igor Bondarenko, Anatoly Makhson, Joerg T. Hartmann, Jorge Aparicio, Filippo de Braud, Serban Donea, Heinz Ludwig, Gunter Schuch, Christopher Stroh, Anja H. Loos, Angela Zubel, and Piotr Koralewski

\section{$\begin{array}{llllllll}\text { A } & \text { B } & \text { S } & \text { T } & \text { R } & \text { A } & \text { C } & \text { T }\end{array}$}

\section{Purpose}

This randomized study assessed whether the best overall response rate (ORR) of cetuximab combined with oxaliplatin, leucovorin, and fluorouracil (FOLFOX-4) was superior to that of FOLFOX-4 alone as first-line treatment for metastatic colorectal cancer. The influence of KRAS mutation status was investigated.

\section{Patients and Methods}

Patients received cetuximab $\left(400 \mathrm{mg} / \mathrm{m}^{2}\right.$ initial dose followed by $250 \mathrm{mg} / \mathrm{m}^{2} / \mathrm{wk}$ thereafter) plus FOLFOX-4 (oxaliplatin $85 \mathrm{mg} / \mathrm{m}^{2}$ on day 1, plus leucovorin $200 \mathrm{mg} / \mathrm{m}^{2}$ and fluorouracil as a 400 $\mathrm{mg} / \mathrm{m}^{2}$ bolus followed by a $600 \mathrm{mg} / \mathrm{m}^{2}$ infusion during 22 hours on days 1 and $2 ; \mathrm{n}=169$ ) or FOLFOX-4 alone ( $n=168$ ). Treatment was continued until disease progression or unacceptable toxicity. KRAS mutation status was assessed in the subset of patients with assessable tumor samples $(n=233)$.

\section{Results}

The confirmed ORR for cetuximab plus FOLFOX-4 was higher than with FOLFOX-4 alone $(46 \% v$ $36 \%)$. A statistically significant increase in the odds for a response with the addition of cetuximab to FOLFOX-4 could not be established (odds ratio $=1.52 ; P=.064$ ). In patients with KRAS wild-type tumors, the addition of cetuximab to FOLFOX-4 was associated with a clinically significant increased chance of response (ORR $=61 \% \vee 37 \%$; odds ratio $=2.54 ; P=.011$ ) and a lower risk of disease progression (hazard ratio $=0.57 ; P=.0163$ ) compared with FOLFOX-4 alone. Cetuximab plus FOLFOX-4 was generally well tolerated.

\section{Conclusion}

KRAS mutational status was shown to be a highly predictive selection criterion in relation to the treatment decision regarding the addition of cetuximab to FOLFOX-4 for previously untreated patients with metastatic colorectal cancer.

\section{J Clin Oncol 27:663-671. (c) 2008 by American Society of Clinical Oncology}

\section{INTRODUCTION}

The epidermal growth factor receptor (EGFR) is a clinically validated anticancer molecular target that is expressed in the majority of colorectal tumors. ${ }^{1-5}$ Cetuximab (Erbitux, developed by Merck KGaA [Darmstadt, Germany], under license from Imclone New York, NY]) is an immunoglobulin G1 monoclonal antibody that specifically targets EGFR with high affinity, competitively inhibiting endogenous ligand binding and liganddependent downstream signaling. ${ }^{6,7}$ Binding to the tumor cell also initiates antibody-dependent cell-mediated cytotoxicity. ${ }^{8-10}$ Randomized phase II and III metastatic colorectal cancer (mCRC) studies have demonstrated the efficacy and tolerability of cetuximab, as monotherapy ${ }^{2,11}$ or in combination with irinotecan, ${ }^{2,12}$ after the failure of previous chemotherapy regimens including irinotecan and/or oxaliplatin. Furthermore, in the first-line setting, building on promising phase II data, ${ }^{13}$ the phase III Cetuximab Combined With Irinotecan in First-Line Therapy for Metastatic Colorectal Cancer (CRYSTAL) study has shown that the addition of cetuximab to infusional fluorouracil (FU)/leucovorin (LV)/irinotecan (FOLFIRI) significantly improves the progression-free survival (PFS) time, response rate, and $\mathrm{R} 0$ resection rate in $\mathrm{mCRC}$ patients, compared with FOLFIRI alone. ${ }^{14}$ 
Phase II studies examining the activity and tolerability of cetuximab combined with oxaliplatin and FU/LV (FOLFOX-4) have provided encouraging results. ${ }^{15,16}$ To further evaluate this combination, the Oxaliplatin and Cetuximab in First-Line Treatment of mCRC (OPUS) study compared the efficacy and safety of cetuximab plus FOLFOX-4 with FOLFOX-4 alone as first-line therapy for mCRC.

The definition of relevant molecular characteristics of an individual tumor (biomarker evaluation) will increasingly enable the selection of patients most likely to benefit from particular treatments. ${ }^{17}$ A recent subgroup analysis of a randomized phase III study of the immunoglobulin G2 EGFR-targeting monoclonal antibody panitumumab in patients with chemotherapy-refractory mCRC suggested a lack of clinical activity in patients whose tumors had activating mutations at codon 12 or 13 of the KRAS gene. ${ }^{18}$ Small retrospective studies in chemotherapy-refractory patients have also suggested that clinical responses to cetuximab are confined to those mCRC patients (approximately 55\% to 70\%) whose tumors are wild type for KRAS. ${ }^{19-22}$ This assertion has recently been confirmed in subgroup analyses of the randomized phase III CRYSTAL and CO.17 studies. ${ }^{23,24}$ A subgroup analysis of the OPUS study therefore investigated whether tumor KRAS mutation status was predictive of a clinical response to cetuximab plus FOLFOX-4.

\section{PATIENTS AND METHODS}

\section{Patient Eligibility}

Patients were eligible for inclusion if they were $\geq 18$ years old; had a histologically confirmed, first-occurrence of a nonresectable, EGFRexpressing mCRC with at least one radiologically measurable lesion; a life expectancy of $\geq 12$ weeks; an Eastern Cooperative Oncology Group Performance Status (ECOG PS) $\leq 2$; and adequate hepatic, renal, and bone marrow function. Patients were ineligible if they were pregnant, had a history of previous exposure to EGFR-targeted therapy or previous chemotherapy (except adjuvant treatment) for mCRC, or had uncontrolled severe organ or metabolic dysfunction. The study was approved by relevant ethics committees and was conducted in accordance with the Declaration of Helsinki. All patients provided written, informed consent.

\section{Study Design}

This was an open-label, randomized, multicenter phase II study comparing the efficacy and safety of cetuximab combined with FOLFOX-4 versus FOLFOX-4 alone in the first-line treatment of EGFR-expressing mCRC. EGFR expression was determined using a DakoCytomation pharmDx immunohistochemistry kit (Dako, Glostrup, Denmark), with one tumor cell staining to any intensity being the minimum criterion required to confirm expression. Randomization (1:1) was carried out using a stratified permutedblock procedure, with ECOG PS ( 0 and $1 v 2)$ as a stratification factor.

On day 1 of a 14-day treatment cycle, patients received cetuximab (initial dose $400 \mathrm{mg} / \mathrm{m}^{2}$ infused during 2 hours, and $250 \mathrm{mg} / \mathrm{m}^{2}$ weekly during 1 hour thereafter) followed after 1 hour by FOLFOX-4 (oxaliplatin $85 \mathrm{mg} / \mathrm{m}^{2}$ on day 1 , infused during 2 hours; LV $200 \mathrm{mg} / \mathrm{m}^{2}$, infused during 2 hours, followed by FU as a $400 \mathrm{mg} / \mathrm{m}^{2}$ intravenous bolus then a 600 $\mathrm{mg} / \mathrm{m}^{2}$ infusion during 22 hours on days 1 and 2) or FOLFOX-4 alone, until the occurrence of progressive disease (PD) or unacceptable toxicity. In the case of unacceptable toxicity or intolerance to FU/LV, oxaliplatin, or cetuximab, the agent responsible could be stopped and the patient could continue with the other study medication(s). Radiologic assessment of response was carried out every 8 weeks until PD or withdrawal for any reason.

The primary objective was to assess whether the best confirmed overall response rate of cetuximab plus FOLFOX-4 was superior to that of FOLFOX-4 alone. Tumor response including disease progression was assessed by an independent review committee according to modified WHO criteria. The independent review committee conducted a blinded review of images and clinical data using a common set of prespecified criteria. The data cutoff for the primary analysis of response was chosen to be 20 weeks after the last randomly assigned patient started study treatment (August 4, 2006) so that all patients could have at least two 8-week evaluations. Secondary objectives included treatment comparisons of the rate of curative metastatic surgery, duration of response, disease control rate, PFS time, OS time, and safety, all of which were based on data from a longer follow-up time (March 1, 2007). A retrospective subgroup analysis investigated associations between PFS and response rate and the KRAS mutation status of tumors.

\section{Statistical Methods and Considerations}

The primary efficacy analysis was performed on the intention-totreat (ITT) population, defined as all randomly assigned patients who received at least one dose of study treatment. Assuming a difference in the rate of best confirmed response of at least $20 \%$ between the two treatments (ie, approximately $70 \%$ with cetuximab plus FOLFOX-4 and 50\% with FOLFOX- 4 alone for the stratum with ECOG PS 0 to 1, and $66 \%$ and $45 \%$, respectively, for the ECOG PS 2 stratum), the Cochran-Mantel-Haenszel $(\mathrm{CMH})^{25,26}$ odds ratio (OR) over the strata was expected to be 2.33. A sample size of approximately 146 patients per group was therefore calculated as necessary to detect an odds ratio of 2.33 at level $\alpha=.05$, with a

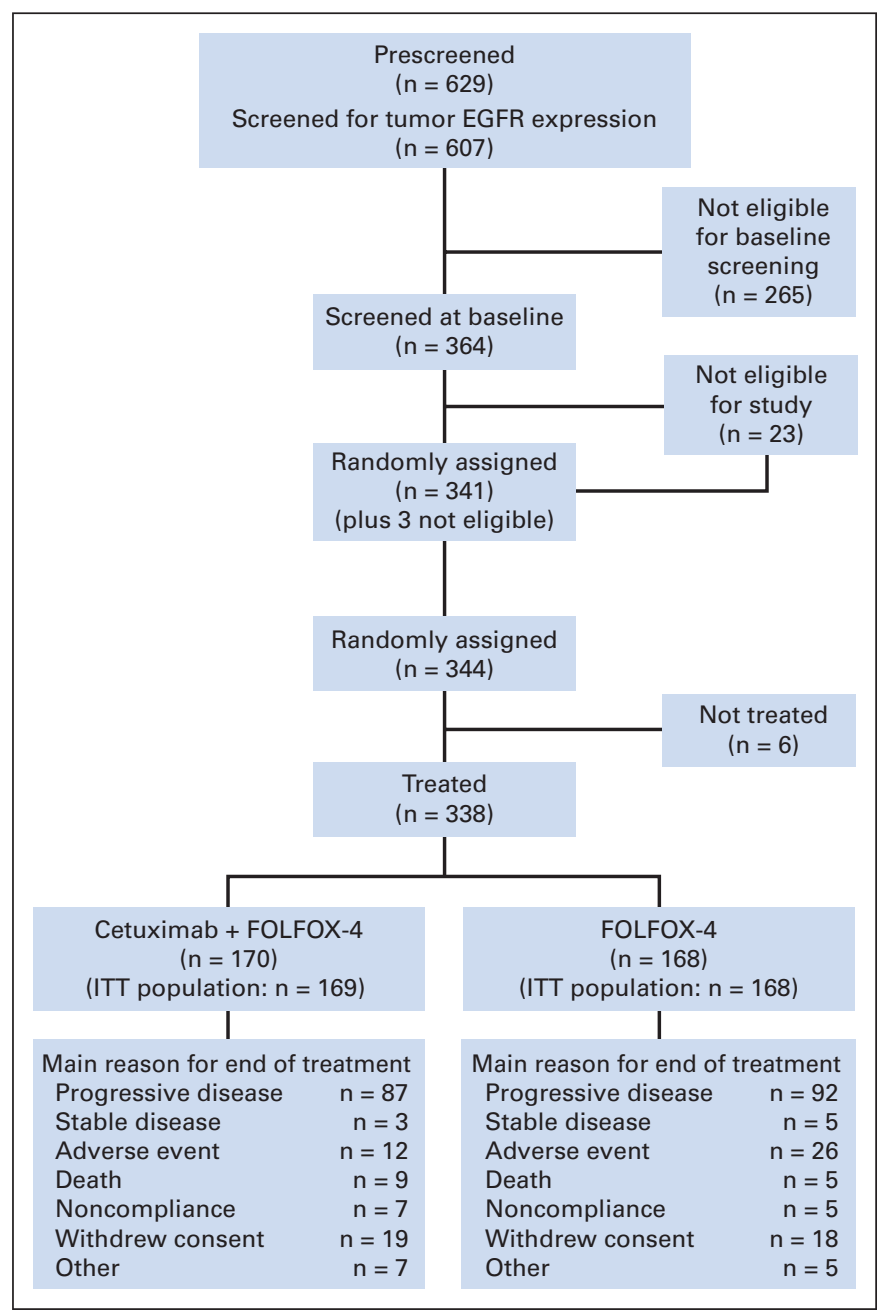

Fig 1. Disposition of subjects at the time of the data cutoff March 1, 2007 EGFR, epidermal growth factor receptor; FOLFOX-4, oxaliplatin, leucovorin, and fluorouracil; ITT, intention to treat. 
power of $90 \%$. With the exception of the test of the null hypothesis of $\mathrm{OR}=1$ over the entire sample population, all further efficacy analyses were exploratory and $P$ values were not adjusted for the multiplicity of statistical tests. Time-to-event data were analyzed using the Kaplan-Meier method. ${ }^{27}$ All analyses were repeated by KRAS mutation status in those ITT patients whose tumors were available and provided an assessable DNA sample (KRAS population).

\section{KRAS Mutation Detection}

Tumor DNA was extracted from formaldehyde-fixed paraffin-embedded tissues, and screened for the presence of KRAS codon 12 and 13 mutations using a polymerase chain reaction (PCR) clamping and melting curve technique adapted from Chen et $\mathrm{al}^{28}$ (full methods information is listed in the Appendix, online only). Briefly, a one-step Lightcycler PCR reaction (LightMix, k-ras Gly12; TIB MOLBIOL, Berlin, Germany) incorporated a codon 12

\begin{tabular}{|c|c|c|c|c|c|c|c|c|c|c|c|c|c|c|c|c|}
\hline \multirow[b]{2}{*}{ Characteristic } & \multicolumn{2}{|c|}{$\begin{array}{c}\text { FOLFOX-4 } \\
(\mathrm{n}=168)\end{array}$} & \multicolumn{2}{|c|}{$\begin{array}{c}\text { Cetuximab + } \\
\text { FOLFOX-4 } \\
(\mathrm{n}=169)\end{array}$} & \multicolumn{2}{|c|}{$\begin{array}{c}\text { FOLFOX-4 } \\
(\mathrm{n}=120)\end{array}$} & \multicolumn{2}{|c|}{$\begin{array}{c}\text { Cetuximab + } \\
\text { FOLFOX-4 } \\
(\mathrm{n}=113)\end{array}$} & \multicolumn{2}{|c|}{$\begin{array}{c}\text { FOLFOX-4 } \\
(n=73)\end{array}$} & \multicolumn{2}{|c|}{$\begin{array}{c}\text { Cetuximab }+ \\
\text { FOLFOX-4 } \\
(\mathrm{n}=61) \\
\end{array}$} & \multicolumn{2}{|c|}{$\begin{array}{c}\text { FOLFOX-4 } \\
(n=47)\end{array}$} & \multicolumn{2}{|c|}{$\begin{array}{c}\text { Cetuximab }+ \\
\text { FOLFOX-4 } \\
(\mathrm{n}=52) \\
\end{array}$} \\
\hline & $\begin{array}{c}\text { No. of } \\
\text { Patients }\end{array}$ & $\%$ & $\begin{array}{c}\text { No. of } \\
\text { Patients }\end{array}$ & $\%$ & $\begin{array}{l}\text { No. of } \\
\text { Patients }\end{array}$ & $\%$ & $\begin{array}{l}\text { No. of } \\
\text { Patients }\end{array}$ & $\%$ & $\begin{array}{c}\text { No. of } \\
\text { Patients }\end{array}$ & $\%$ & $\begin{array}{c}\text { No. of } \\
\text { Patients }\end{array}$ & $\%$ & $\begin{array}{l}\text { No. of } \\
\text { Patients }\end{array}$ & $\%$ & $\begin{array}{c}\text { No. of } \\
\text { Patients }\end{array}$ & $\%$ \\
\hline Female & 76 & 45 & 80 & 47 & 54 & 45 & 56 & 50 & 29 & 40 & 31 & 51 & 25 & 53 & 25 & 48 \\
\hline \multicolumn{17}{|l|}{ Age, years } \\
\hline Median & \multicolumn{2}{|c|}{60} & \multicolumn{2}{|c|}{62} & \multicolumn{2}{|l|}{60} & 59 & & 59 & & 59 & & 61 & & 60 & \\
\hline Range & $30-8$ & & $24-8$ & & $30-82$ & & $24-82$ & & $36-82$ & & $24-7$ & & $30-75$ & & $41-82$ & \\
\hline$<65$ & 109 & 65 & 96 & 57 & 77 & 64 & 70 & 62 & 46 & 63 & 39 & 64 & 31 & 66 & 31 & 60 \\
\hline$\geq 65$ & 59 & 35 & 73 & 43 & 43 & 63 & 43 & 38 & 27 & 37 & 22 & 36 & 16 & 34 & 21 & 40 \\
\hline ROW & 1 & 1 & 0 & & 0 & & 0 & & 0 & & 0 & & 0 & & 0 & \\
\hline ECOG PS at baseline & & & & & & & & & & & & & & & & \\
\hline 0 & 75 & 45 & 65 & 39 & 50 & 42 & 34 & 30 & 27 & 37 & 19 & 31 & 23 & 49 & 15 & 29 \\
\hline 1 & 76 & 45 & 89 & 53 & 56 & 47 & 70 & 62 & 37 & 51 & 37 & 61 & 19 & 40 & 33 & 63 \\
\hline 2 & 17 & 10 & 15 & 9 & 14 & 12 & 9 & 8 & 9 & 12 & 5 & 8 & 5 & 11 & 4 & 8 \\
\hline Disease duration, months & & & & & & & & & & & & & & & & \\
\hline Median, CRC & 2.2 & & 2.1 & & 2.1 & & 2.2 & & 2.0 & & 2.1 & & 2.1 & & 2.3 & \\
\hline Q1-03 & $1.3-1$ & & $1.2-9$ & & $1.3-14$ & & $1.4-10$ & & $1.3-15$ & & $1.2-9$ & & $1.2-7$ & & $1.6-10$ & \\
\hline Median, mCRC & 1.5 & & 1.4 & & 1.6 & & 1.4 & & 1.6 & & 1.3 & & 1.4 & & 1.6 & \\
\hline Q1-03 & $1.1-2$ & & $0.9-2$ & & $1.1-2$ & & $1.0-2$ & & $1.2-2$ & & $0.9-2$ & & $1.1-2$ & & $1.0-2$ & \\
\hline Tumor site & & & & & & & & & & & & & & & & \\
\hline Colon & 89 & 53 & 92 & 54 & 66 & 55 & 61 & 54 & 41 & 56 & 31 & 51 & 25 & 53 & 30 & 58 \\
\hline Organs with metastases & & & & & & & & & & & & & & & & \\
\hline 0 & $1^{*}$ & 0.6 & 0 & & 0 & & 0 & & 0 & & 0 & & 0 & & 0 & \\
\hline 1 & 69 & 41 & 74 & 44 & 52 & 43 & 50 & 44 & 31 & 42 & 32 & 52 & 21 & 45 & 18 & 35 \\
\hline 2 & 63 & 38 & 60 & 36 & 41 & 34 & 38 & 34 & 24 & 33 & 17 & 28 & 17 & 36 & 21 & 40 \\
\hline 3 & 18 & 11 & 22 & 13 & 15 & 13 & 16 & 14 & 12 & 16 & 9 & 15 & 3 & 6 & 7 & 13 \\
\hline$\geq 4$ & 17 & 10 & 13 & 8 & 12 & 10 & 9 & 8 & 6 & 8 & 3 & 5 & 6 & 13 & 6 & 12 \\
\hline Prior therapy ${ }^{\dagger}$ & & & & & & & & & & & & & & & & \\
\hline Surgery & 152 & 91 & 137 & 81 & 108 & 90 & 100 & 88 & 67 & 92 & 50 & 82 & 41 & 87 & 50 & 96 \\
\hline Adjuvant chemotherapy & 36 & 21 & 21 & 12 & 19 & 16 & 14 & 12 & 12 & 16 & 8 & 13 & 7 & 15 & 6 & 12 \\
\hline Radiotherapy & 23 & 14 & 18 & 11 & 13 & 11 & 13 & 12 & 9 & 12 & 5 & 8 & 4 & 9 & 8 & 15 \\
\hline
\end{tabular}


to 14 spanning locked nucleic acid oligomer to suppress amplification of the wild-type sequence. ${ }^{29}$ Post-PCR hybridization and melting curve analysis using fluorescently tagged oligonucleotides incorporated in the original PCR reaction permitted the identification and discrimination of distinct KRAS codon 12 and 13 missense mutations.

\section{Safety Analysis}

The safety population comprised all patients who received at least one dose of study treatment. All adverse events (AEs) reported with onset on or after the first day of study medication and captured up to 6 weeks after the end of the last administration of study treatment were coded using the Medical Dictionary for Regulatory Activities version 8.1 and summarized by worst severity per patient according to the National Cancer Institute Common Toxicity Criteria version 2.0.

\section{RESULTS}

\section{Patient Demographics}

Three hundred forty-four patients were randomly assigned (three of them in error). Of the eligible patients, 337 received study treatment at 79 centers. Study accrual lasted from July 2005 until March 2006, with 169 patients receiving cetuximab plus FOLFOX-4 and 168 patients receiving FOLFOX-4 alone (Fig 1). Baseline demographic and disease characteristics were generally well balanced between the treatment groups (Table 1). DNA suitable for KRAS mutation analysis was extracted from the tumor samples of 233 patients, 113 and 120 of whom had received cetuximab plus FOLOFOX-4 and FOLFOX-4 alone, respectively. These patients comprised the KRAS population. In general, the population with tissue available for KRAS analysis $(\mathrm{n}=233)$ was considered to be representative of the overall ITT population $(\mathrm{n}=337)$ in relation to demographic parameters (Table 1).

\section{Treatment Compliance}

The median duration of cetuximab treatment was 24 weeks, with $84 \%$ of patients having a relative dose-intensity (RDI) of $\geq 80 \%$. Similar numbers of patients in both arms had RDIs of $\geq 80 \%$ for oxaliplatin $(75 \%$ and $80 \%$ of patients receiving cetuximab plus

\begin{tabular}{|c|c|c|c|c|c|c|c|c|c|c|c|c|c|c|c|c|}
\hline \multirow[b]{3}{*}{ Characteristic } & \multicolumn{4}{|c|}{ ITT Population ( $\mathrm{n}=337$ ) } & \multicolumn{4}{|c|}{ KRAS Population ( $\mathrm{n}=233$ ) } & \multicolumn{4}{|c|}{ KRAS Wild Type $(\mathrm{n}=134)$} & \multicolumn{4}{|c|}{ KRAS Mutant $(\mathrm{n}=99)$} \\
\hline & \multicolumn{2}{|c|}{$\begin{array}{c}\text { FOLFOX-4 } \\
(n=168)\end{array}$} & \multicolumn{2}{|c|}{$\begin{array}{c}\text { Cetuximab + } \\
\text { FOLFOX-4 } \\
(n=169)\end{array}$} & \multicolumn{2}{|c|}{$\begin{array}{c}\text { FOLFOX-4 } \\
(n=120)\end{array}$} & \multicolumn{2}{|c|}{$\begin{array}{c}\text { Cetuximab }+ \\
\text { FOLFOX-4 } \\
(\mathrm{n}=113)\end{array}$} & \multicolumn{2}{|c|}{$\begin{array}{c}\text { FOLFOX-4 } \\
(n=73)\end{array}$} & \multicolumn{2}{|c|}{$\begin{array}{c}\text { Cetuximab + } \\
\text { FOLFOX-4 } \\
(\mathrm{n}=61)\end{array}$} & \multicolumn{2}{|c|}{$\begin{array}{c}\text { FOLFOX-4 } \\
(n=47)\end{array}$} & \multicolumn{2}{|c|}{$\begin{array}{c}\text { Cetuximab + } \\
\text { FOLFOX-4 } \\
(n=52) \\
\end{array}$} \\
\hline & $\begin{array}{l}\text { No. of } \\
\text { Patients }\end{array}$ & $\%$ & $\begin{array}{l}\text { No. of } \\
\text { Patients }\end{array}$ & $\%$ & $\begin{array}{l}\text { No. of } \\
\text { Patients }\end{array}$ & $\%$ & $\begin{array}{l}\text { No. of } \\
\text { Patients }\end{array}$ & $\%$ & $\begin{array}{l}\text { No. of } \\
\text { Patients }\end{array}$ & $\%$ & $\begin{array}{l}\text { No. of } \\
\text { Patients }\end{array}$ & $\%$ & $\begin{array}{l}\text { No. of } \\
\text { Patients }\end{array}$ & $\%$ & $\begin{array}{l}\text { No. of } \\
\text { Patients }\end{array}$ & $\%$ \\
\hline \multicolumn{17}{|l|}{ Best overall response* } \\
\hline Complete response & 1 & 0.6 & 2 & 1 & 3 & 3 & 2 & 2 & 1 & 1 & 2 & 3 & 2 & 4 & 0 & \\
\hline Partial response & 59 & 35 & 75 & 44 & 47 & 39 & 52 & 46 & 26 & 36 & 35 & 57 & 21 & 45 & 17 & 33 \\
\hline Stable disease & 76 & 45 & 67 & 40 & 47 & 39 & 46 & 41 & 30 & 41 & 19 & 31 & 17 & 36 & 27 & 52 \\
\hline Progressive disease & 21 & 13 & 18 & 11 & 17 & 14 & 10 & 9 & 12 & 16 & 3 & 5 & 5 & 11 & 7 & 13 \\
\hline Not assessable & 11 & 7 & 7 & 4 & 6 & 5 & 3 & 3 & 4 & 5 & 2 & 3 & 2 & 4 & 1 & 2 \\
\hline \multicolumn{17}{|l|}{$\begin{array}{l}\text { Best overall response } \\
\text { ratet, \% }\end{array}$} \\
\hline All patients & \multicolumn{2}{|l|}{36} & \multicolumn{2}{|l|}{46} & \multicolumn{2}{|l|}{42} & \multicolumn{2}{|l|}{48} & \multicolumn{2}{|l|}{37} & \multicolumn{2}{|l|}{61} & \multicolumn{4}{|c|}{33} \\
\hline $95 \% \mathrm{Cl}$ & \multicolumn{2}{|l|}{$29-44$} & \multicolumn{2}{|l|}{$38-53$} & $33-51$ & & $38-57$ & & $26-49$ & & $47-73$ & & $34-64$ & & $20-47$ & \\
\hline Odds ratio & & 1.5 & & & & 1.31 & & & & 2.54 & & & & 0.5 & & \\
\hline $95 \% \mathrm{Cl}$ & & 0.975 & 2.355 & & & 0.779 & -2.208 & & & 1.238 & -5.227 & & & 223 & 1.150 & \\
\hline$P$ (stratified $\mathrm{CMH}$ test) & & .0 & & & & .30 & & & & .01 & 11 & & & & & \\
\hline Disease control rate $\neq, \%$ & 81 & & 85 & & 81 & & 89 & & 78 & & 92 & & 85 & & 85 & \\
\hline $95 \% \mathrm{Cl}$ & $74-87$ & & $79-90$ & & $73-87$ & & $81-94$ & & $67-87$ & & $82-97$ & & $72-94$ & & $72-93$ & \\
\hline PFS time, months & & & & & & & & & & & & & & & & \\
\hline Median & 7.2 & & 7.2 & & 7.2 & & 7.3 & & 7.2 & & 7.7 & & 8.6 & & 5.5 & \\
\hline $95 \% \mathrm{Cl}$ & $6.0-7.8$ & & $5.6-7.7$ & & $6.0-7.9$ & & $5.6-8.1$ & & $5.6-7.4$ & & $7.1-12.0$ & & $6.5-9.5$ & & 4.0-7.4 & \\
\hline Hazard ratio & & 0.9 & & & & 0.92 & & & & 0.57 & & & & 1.8 & & \\
\hline $95 \% \mathrm{Cl}$ & & 0.705 & 1.230 & & & $0.665-$ & -1.295 & & & $0.358-$ & 0.907 & & & .095 & 3.056 & \\
\hline$P$ (log-rank test) & & & 70 & & & & 609 & & & & 163 & & & & 92 & \\
\hline PFS rates, \% & & & & & & & & & & & & & & & & \\
\hline 3 months & 85 & & 83 & & 82 & & 86 & & 78 & & 93 & & 87 & & 78 & \\
\hline $95 \% \mathrm{Cl}$ & $79-90$ & & $78-89$ & & $75-89$ & & $80-93$ & & $69-88$ & & $87-100$ & & $77-97$ & & $67-90$ & \\
\hline 6 months & 59 & & 53 & & 60 & & 54 & & 54 & & 66 & & 69 & & 39 & \\
\hline $95 \% \mathrm{Cl}$ & $51-68$ & & $45-61$ & & $50-69$ & & $44-64$ & & $42-66$ & & $53-79$ & & $54-83$ & & $25-54$ & \\
\hline 9 months & 34 & & 34 & & 33 & & 35 & & 27 & & 47 & & 42 & & 20 & \\
\hline $95 \% \mathrm{Cl}$ & $26-43$ & & $26-43$ & & $23-43$ & & $25-45$ & & $15-39$ & & $33-62$ & & $24-61$ & & $7-33$ & \\
\hline 12 months & 12 & & 24 & & 13 & & 20 & & 13 & & 30 & & 14 & & 6 & \\
\hline $95 \% \mathrm{Cl}$ & $3-20$ & & $15-33$ & & $4-23$ & & $8-32$ & & $2-24$ & & $10-49$ & & $0-30$ & & $0-16$ & \\
\hline $\begin{array}{l}\text { NOTE. } P<0.05 \text { for bold } \\
\text { Abbreviations: ITT, intenti } \\
{ }^{*} \text { As assessed by independ } \\
\text { †Complete response }+ \\
\neq \text { Complete response }+\end{array}$ & $\begin{array}{l}\text { ues. } \\
\text { to treat; F } \\
\text { t review. } \\
\text { al respons } \\
\text { al respons }\end{array}$ & $\begin{array}{l}\text { FOLFC } \\
\text { Best o } \\
\text { ise. } \\
\text { ise + }\end{array}$ & $\begin{array}{l}\text { X-4, oxalipl } \\
\text { verall respor } \\
\text { stable diseas }\end{array}$ & $\begin{array}{l}\text { atin, } \\
\text { nse o } \\
\text { se. }\end{array}$ & $\begin{array}{l}\text { eucovorin, a } \\
\text { the ITT pop }\end{array}$ & $\begin{array}{l}\text { and } \mathrm{fl} \\
\text { pulati }\end{array}$ & $\begin{array}{l}\text { luorouracil; } \\
\text { ion was base }\end{array}$ & $\begin{array}{l}\mathrm{CMH} \\
\text { ed on }\end{array}$ & $\begin{array}{l}\text { Cochran-M } \\
\text { data availab }\end{array}$ & $\begin{array}{l}\text { lantel- } \\
\text { ole at }\end{array}$ & $\begin{array}{l}\text {-Haenszel; P } \\
\text { the a priori fi> }\end{array}$ & xed & $\begin{array}{l}\text { rogression- } f \\
\text { me of the co }\end{array}$ & $\begin{array}{l}\text { free } \\
\text { onfirr }\end{array}$ & $\begin{array}{l}\text { urvival. } \\
\text { natory an }\end{array}$ & \\
\hline
\end{tabular}


FOLFOX-4 and FOLFOX-4, respectively) and FU (67\% and 70\% of patients, respectively). Reductions and delays in cetuximab dosing were primarily because of skin reactions, and delays in chemotherapy dosing were because of hematologic, GI, or neurologic reactions. In the KRAS population, comparable levels of exposure were reached; $86 \%$ of patients who received cetuximab had a RDI $\geq 80 \%$. RDIs of $\geq 80 \%$ were also reported for $73 \%$ and $81 \%$ of patients receiving oxaliplatin and for $65 \%$ and $72 \%$ of patients receiving $\mathrm{FU}$ in the cetuximab plus FOLFOX-4 and FOLFOX-4 arms, respectively.

\section{Efficacy}

Efficacy data are summarized in Table 2. The best confirmed overall response rate in the cetuximab plus FOLFOX-4 group was $46 \%$, and in the FOLFOX-4-alone group, this value was $36 \%$. The comparison between the two treatment groups resulted in a common ECOG PS-adjusted OR of 1.52 (95\% CI, 0.98 to 2.36), indicating an increase in the chance of a response in the cetuximab plus FOLFOX-4 arm by $52 \%$. This increase was not statistically significant at the 5\% level (ECOG PS-adjusted CMH test, $P=.064$ ). A slightly greater treatment effect was observed in the group of participants $(\mathrm{n}=153 v 152)$ with ECOG PS 0 to $1(\mathrm{OR}=1.65 ; 95 \% \mathrm{CI}$, 1.04 to 2.60 ; $\mathrm{CMH}$ test, $P=.032$ ). Regarding the secondary efficacy end points, although a marked benefit in relation to PFS time was not detected (Table 2; Fig 2A), the addition of cetuximab to FOLFOX-4 was associated with an approximate doubling of the R0 resection rate from $2.4 \%$ (four of 168 patients) for FOLFOX-4 alone to $4.7 \%$ (eight of 169 patients) for cetuximab plus FOLFOX-4. The median duration of response was also longer in the cetuximab group (9.0 $v 5.7$ months).

KRAS mutations in codons 12/13 (hereinafter called KRAS mutations) were detected in the tumor tissue of 99 of 233 (42\%) patients, 52 of whom received cetuximab plus FOLFOX-4 and 47 of whom received FOLFOX-4 alone (Table 2). No mutations were detected in the tumors of 134 patients (58\%; hereinafter called KRAS wild-type tumors), 61 of whom received cetuximab plus
FOLFOX-4 and 73 of whom received FOLFOX-4 alone. In patients whose tumors were scored as KRAS wild type, the addition of cetuximab to FOLFOX-4 was associated with a 2.54-fold increased chance for response compared with FOLFOX-4 alone (61\% v 37\%; $\mathrm{OR}=2.54 ; 95 \% \mathrm{CI}, 1.24$ to 5.23 ; ECOG PS-adjusted CMH test, $P=.011)$. Conversely, for patients with tumor KRAS mutations, the response rate for cetuximab plus FOLFOX-4 was lower, compared with FOLFOX-4 alone (33\% v 49\%; OR = 0.51; 95\% CI, 0.22 to 1.15 ; ECOG PS-adjusted CMH test, $P=.106)$. The R0 resection rate was more than doubled in patients with KRAS wild-type tumors who received cetuximab plus FOLFOX-4 (six of 61 patients; 9.8\%) compared with those who received FOLFOX-4 alone (three of 73 patients; $4.1 \%$ ). In contrast, in patients whose tumors carried KRAS mutations, R0 resection rates were similar in both treatment arms (one in 52 and one in 47 patients, respectively; $1.9 \%$ and $2.1 \%$ ). However, due to the small patient numbers, these data have to be interpreted with caution.

The risk of disease progression (Figs $2 \mathrm{~A}$ and $2 \mathrm{~B}$ ) was similar for both the ITT (cetuximab plus FOLFOX- $4 v$ FOLFOX-4 alone; hazard ratio $[\mathrm{HR}]=0.93$; log-rank test, $P=.6170$ ) and the KRAS population $(\mathrm{HR}=0.93$; log-rank test, $P=.6609)$, adding additional evidence consistent with the representability of the KRAS population. A marked risk reduction for progression was evident between the study arms when comparing patients according to whether KRAS mutations were detected in their tumor DNA. Specifically, as summarized in Table 2 and Figure 3, the addition of cetuximab to FOLFOX-4 was associated with a $43 \%$ reduction in the risk of progression in patients with KRAS wild-type tumors (Fig 3C; $\mathrm{HR}=0.57$; log-rank test, $P=.0163)$. In those patients receiving cetuximab plus FOLFOX-4, the risk of disease progression was lower for those whose tumors were wild type for KRAS, compared with those whose tumors carried mutations (Fig 3A; HR $=0.45$; log-rank test, $P=.0009$ ). However, in patients whose tumors carried mutations of KRAS, the risk of disease progression was found to be lower for those receiving FOLFOX-4 alone compared with those receiving cetuximab plus FOLFOX-4 (Fig 3D;

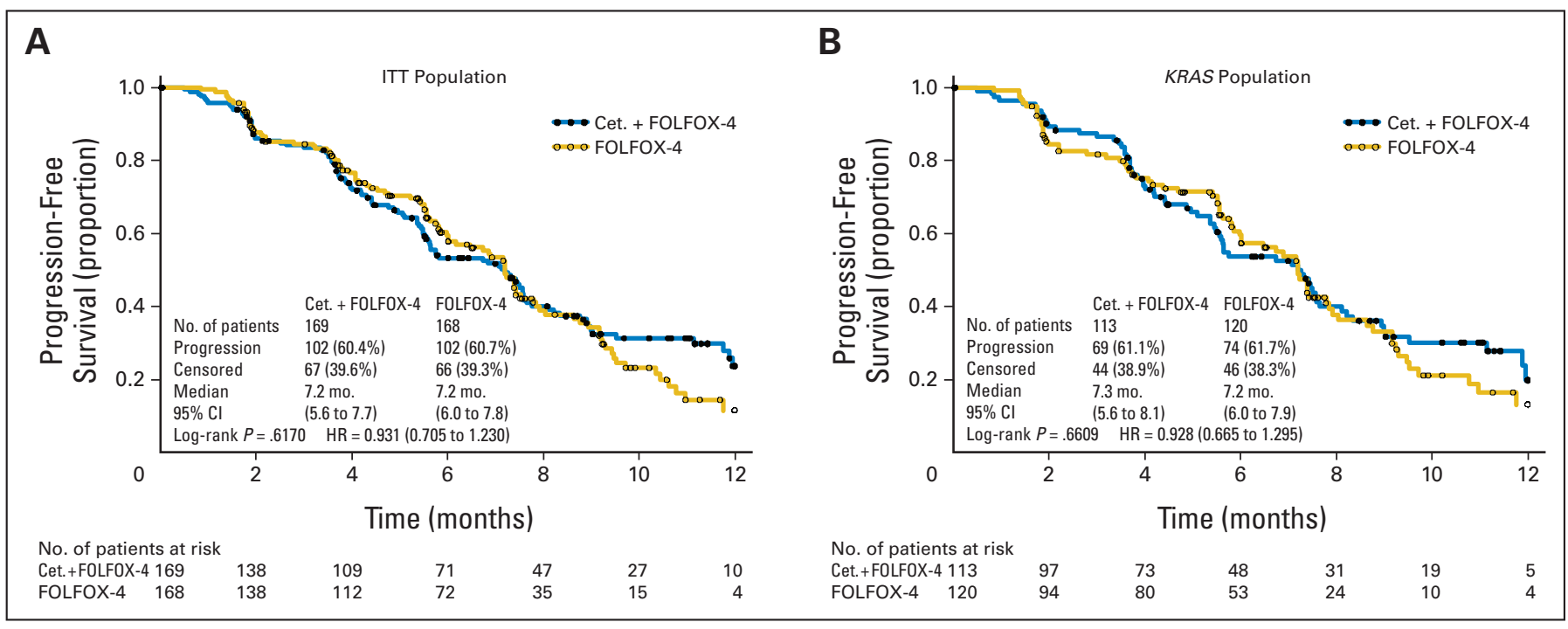

Fig 2. Kaplan and Meier plots for progression-free survival time for patients in each study arm in (A) the intention to treat (ITT) population and (B) the KRAS population. Cet., cetuximab; FOLFOX-4, oxaliplatin, leucovorin, and fluorouracil; mo., months. 


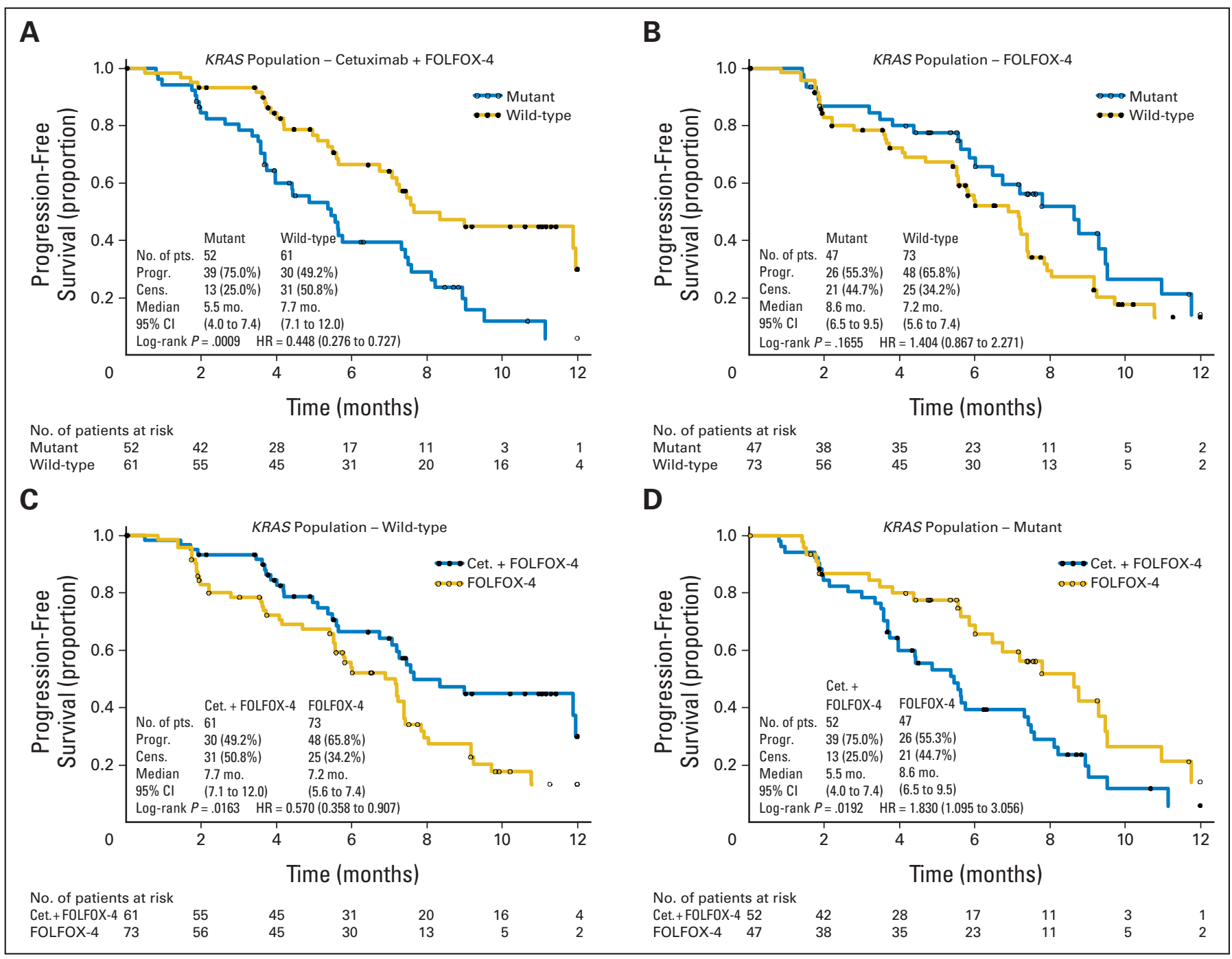

Fig 3. Kaplan and Meier plots for progression-free survival time according to tumor KRAS mutation status in patients (pts) who received (A) cetuximab (Cet.) plus oxaliplatin, leucovorin, and fluorouracil (FOLFOX-4) or (B) FOLFOX-4 alone and according to treatment arm for pts whose tumors were (C) wild type or (D) mutant for KRAS. Progr., progression; Cens., censored; HR, hazard ratio.

$\mathrm{HR}=1.83$; log-rank test, $P=.0192$ ). For patients receiving FOLFOX-4 alone, a trend for reduced risk of disease progression associated with tumor KRAS mutation was also apparent (Fig 3B; $\mathrm{HR}=1.40$; log-rank test, $P=.1655)$.

\section{Safety}

The most common AEs at any grade were skin and subcutaneous tissue disorders (90\%) and GI disorders (78\%) in the cetuximab plus FOLFOX-4 group, and GI disorders (73\%) and myelotoxicity (71\%) in the FOLFOX-4-alone group. The most common grade 3/4 AEs were neutropenia (30\% $v 34 \%)$, rash $(11 \% v 0.6 \%)$, and diarrhea $(8 \% v 7 \%)$ respectively, for the cetuximab plus FOLFOX-4 versus the FOLFOX-4- alone group (Table 3).

In the cetuximab plus FOLFOX-4 group, cetuximab was discontinued in 39 patients $(23 \%)$, chemotherapy was discontinued in 51 patients $(30 \%)$, and both were discontinued in 16 patients (9\%) due to AEs. Chemotherapy was discontinued in 42 patients $(25 \%)$ in the FOLFOX-4-alone group due to AEs. The most com- mon reasons for cetuximab discontinuation were hypersensitivity reactions (4\%) and rash (4\%), in accordance with guidelines prespecified in the protocol, and for chemotherapy discontinuation in the cetuximab plus FOLFOX-4 group, neutropenia (4\%) and neuropathy $(3 \%)$. In the FOLFOX-4-alone group, peripheral sensory neuropathy (5\%), neuropathy and paresthesia (4\%), and neutropenia (2\%) were the most common reasons for treatment discontinuation.

At the time of analysis, 111 patients had died; 60 patients (35\%) in the cetuximab plus FOLFOX-4 group and 51 patients $(30 \%)$ in the FOLFOX-4-alone group, mainly due to PD (94 patients). No deaths were related to cetuximab.

\section{DISCUSSION}

The rationale for testing cetuximab in combination with FOLFOX-4 rested on preclinical data suggesting that cetuximab improved the antitumor activity of oxaliplatin and also on promising clinical phase II study data. ${ }^{16,30}$ The confirmed best overall response rate 


\begin{tabular}{|c|c|c|c|c|}
\hline \multirow[b]{2}{*}{ Grade 3/4 Adverse Event } & \multicolumn{2}{|c|}{$\begin{array}{l}\text { FOLFOX-4 } \\
(\mathrm{n}=168)\end{array}$} & \multicolumn{2}{|c|}{$\begin{array}{c}\text { Cetuximab }+ \\
\text { FOLFOX-4 } \\
(n=170)\end{array}$} \\
\hline & $\begin{array}{c}\text { No. of } \\
\text { Patients }\end{array}$ & $\%$ & $\begin{array}{c}\text { No. of } \\
\text { Patients }\end{array}$ & $\%$ \\
\hline Any grade $3 / 4$ event & 117 & 70 & 129 & 76 \\
\hline Neutropenia & 57 & 34 & 51 & 30 \\
\hline Rash* & 1 & 0.6 & 19 & 11 \\
\hline Diarrhea* & 12 & 7 & 14 & 8 \\
\hline Leukopenia & 10 & 6 & 12 & 7 \\
\hline Thrombocytopenia & 4 & 2 & 7 & 4 \\
\hline Fatigue* & 5 & 3 & 7 & 4 \\
\hline Palmar-plantar erythrodysesthesia* & 1 & 0.6 & 7 & 4 \\
\hline Peripheral sensory neuropathy* & 12 & 7 & 7 & 4 \\
\hline Anemia* & 4 & 2 & 6 & 4 \\
\hline \multicolumn{5}{|l|}{ Composite categories } \\
\hline Skin reactions* $\dagger$ & 1 & 0.6 & 30 & 18 \\
\hline Infusion-related reactions $\ddagger$ & 3 & 2 & 8 & 5 \\
\hline
\end{tabular}

Abbreviation: FOLFOX-4, oxaliplatin, leucovorin, and fluorouracil.

*No grade 4 reactions.

†The special adverse event category skin reactions included the following Medical Dictionary for Regulatory Activities 8.1 terms: acne, acne pustular, cellulitis, dermatitis acneiform, dry skin, erysipelas, erythema, face edema, folliculitis, growth of eyelashes, hair growth abnormal, hypertrichosis, nail bed infection, nail bed inflammation, nail disorder, nail infection, paronychia, pruritus, rash, rash erythematous, rash follicular, rash generalized, rash macular, rash maculopapular, rash popular, rash pruritic, rash pustular, skin exfoliation, skin hyperpigmentation, skin necrosis, staphylococcal scalded skin syndrome, telangiectasia, wound necrosis, xerosis.

$\ddagger$ The special adverse event category infusion-related reactions included the following Medical Dictionary for Regulatory Activities 8.1 terms: acute respiratory failure, apnea, asthma, bronchial obstruction, bronchospasm, cyanosis, dyspnea, dyspnea at rest, dyspnea exacerbated, dyspnea exertional, hypoxia, orthopnea, respiratory distress, respiratory failure, chills, hyperpyrexia, pyrexia, acute myocardial infarction, angina pectoris, blood pressure decreased, cardiac failure, cardiopulmonary failure, clonus, convulsion, epilepsy, hypotension, infusion related reaction, loss of consciousness, myocardial infarction, myocardial ischemia, shock, sudden death and syncope, occurring on the first day of treatment; and anaphylactic reaction, anaphylactic shock, anaphylactoid reaction, anaphylactoid shock, drug hypersensitivity and hypersensitivity, occurring at any time during treatment.

for the ITT population, as assessed by blinded independent review, was increased by 10 percentage points by the addition of cetuximab to FOLFOX-4, compared with FOLFOX-4 alone (46\% v 36\%). Although this difference showed a clinically relevant and strong trend, a statistically significant increase in the odds for a response with the addition of cetuximab to FOLFOX-4 could not be established $(P=.064)$.

Although there was no apparent difference in PFS time between the ITT population treatment arms, when retrospective efficacy analyses were carried out in the KRAS population according to KRAS mutation status, striking differences were apparent in relation to tumor response and the risk of disease progression. Patients whose tumors were wild type for KRAS had a clinically relevant increase in the chance of response $(61 \% \vee 37 \%$; OR $=2.54 ; P=.011)$ and decrease in the risk of disease progression $(\mathrm{HR}=0.57 ; P=.016)$ in the cetuximab plus FOLFOX-4 arm compared with those in the FOLFOX-4-alone arm. Somewhat unexpected was the observation that patients whose tumors carried a mutation in KRAS were more likely to derive a clinical benefit if treated with FOLFOX-4 alone rather than cetuximab plus FOLFOX-4 (Fig 3D). Similarly, considering patients receiving FOLFOX-4 alone, there was a trend for improved progression-free survival time in patients whose tumors carried a KRAS mutation (Fig 3B).

It remains to be seen whether the apparently better outcome of patients whose tumors carried KRAS mutations who were treated with FOLFOX-4 alone compared with those treated with cetuximab plus FOLFOX-4 was a chance finding, or whether it might have a biologic explanation. Given that a comparable effect was not observed in equivalent patient groups in the CRYSTAL study when cetuximab was added to FOLFIRI, ${ }^{23}$ the type of chemotherapy (platinum based compared with nonplatinum based) may be a factor in any possible interaction. It is interesting to note in this context that the subgroup of genotyped patients in the randomized phase III CAIRO2 study whose tumors had a mutation in the KRAS gene $(\mathrm{n}=196)$ who received capecitabine/oxaliplatin plus bevacizumab $(\mathrm{n}=103)$ had significantly better PFS than those who received the same regimen plus cetuximab $(n=93) .^{31}$

The OPUS and CAIRO2 studies therefore raise the possibility that in relation to tumors with mutations in the KRAS gene, the addition of EGFR-targeted agents to oxaliplatin-based chemotherapy may impair the efficacy of a cytotoxic component of the combined regimen. In addition, a specific interaction between bevacizumab and cetuximab in relation to the treatment of tumors with KRAS mutations cannot be excluded. Experimental studies are therefore warranted to explore such hypothetical treatment effects in KRAS mutant mCRCs with a view to devising novel clinical strategies to allow extension of the benefit of anti-EGFR therapy to patients whose tumors have KRAS mutations.

In line with recent findings from a phase I dose-escalation study of cetuximab with FOLFIRI, ${ }^{32}$ and the first-line data from the phase III CRYSTAL study, ${ }^{23}$ the results of the current analysis confirm published data from previously treated mCRC, regarding restriction of the activity of cetuximab to patients whose tumors are wild type for KRAS. ${ }^{19-22,24}$ The KRAS data also emphasize the fact that the higher clinical benefit associated with cetuximab essentially may be masked, or at the least underestimated, when considering overall populations of unselected patients in randomized studies, as illustrated by the nonsignificant results in response rate and the comparable risk of disease progression in the ITT population.

The combination of cetuximab and FOLFOX-4 was generally well tolerated, and there was no evidence to suggest that cetuximab increased the frequency or severity of the known toxicities of oxaliplatin and FU or vice versa. In addition, the incidence and severity of skin reactions, infusion-related reactions, and mucositis were consistent with the known safety profile of cetuximab.

In conclusion, this study demonstrated that to a clinically meaningful extent, the addition of cetuximab to FOLFOX-4 as first-line treatment for $\mathrm{mCRC}$ increased the overall response rate and reduced the risk of disease progression in patients whose tumors were wild type for KRAS, compared with FOLFOX-4 alone. These data confirm that KRAS mutation status is a powerful predictive factor in relation to the efficacy of cetuximab treatment, and are a clear demonstration of the potential and increasing importance of tumor biomarker analysis as a component of treatment selection in the clinic. 
AUTHORS' DISCLOSURES OF POTENTIAL CONFLICTS OF INTEREST

Although all authors completed the disclosure declaration, the following author(s) indicated a financial or other interest that is relevant to the subject matter under consideration in this article. Certain relationships marked with a " $U$ " are those for which no compensation was received; those relationships marked with a "C" were compensated. For a detailed description of the disclosure categories, or for more information about ASCO's conflict of interest policy, please refer to the Author Disclosure Declaration and the Disclosures of Potential Conflicts of Interest section in Information for Contributors.

Employment or Leadership Position: Christopher Stroh, Merck Serono (C); Anja H. Loos, Merck Serono (C); Angela Zubel, Merck Serono (C) Consultant or Advisory Role: Carsten Bokemeyer, Merck Serono (C) Stock Ownership: None Honoraria: Carsten Bokemeyer, Merck Serono, Sanofi-aventis; Joerg T. Hartmann, Merck Serono Research Funding: Carsten Bokemeyer, Merck Serono; Gunter Schuch, Merck Serono; Christopher Stroh, Merck Serono Expert Testimony: None Other Remuneration: Serban Donea, Merck Serono, Sanofi-aventis

\section{AUTHOR CONTRIBUTIONS}

Conception and design: Carsten Bokemeyer, Angela Zubel

Administrative support: Joerg T. Hartmann, Anja H. Loos

Provision of study materials or patients: Carsten Bokemeyer, Igor

Bondarenko, Anatoly Makhson, Joerg T. Hartmann, Jorge Aparicio, Filippo de Braud, Serban Donea, Heinz Ludwig, Gunter Schuch, Piotr Koralewski

Collection and assembly of data: Igor Bondarenko, Anatoly Makhson, Joerg T. Hartmann, Jorge Aparicio, Christopher Stroh, Piotr Koralewski

Data analysis and interpretation: Carsten Bokemeyer, Anatoly Makhson, Joerg T. Hartmann, Filippo de Braud, Christopher Stroh, Anja H. Loos

Manuscript writing: Carsten Bokemeyer, Joerg T. Hartmann, Christopher Stroh, Anja H. Loos

Final approval of manuscript: Carsten Bokemeyer, Igor Bondarenko, Anatoly Makhson, Joerg T. Hartmann, Jorge Aparicio, Filippo de Braud, Serban Donea, Heinz Ludwig, Gunter Schuch, Christopher Stroh, Anja H. Loos, Angela Zubel, Piotr Koralewski

\section{REFERENCES}

1. Adenis A, Aranda Aguilar E, Robin YM, et al: Expression of the epidermal growth factor receptor (EGFR or HER1) and human epidermal growth factor receptor 2 (HER2) in a large scale metastatic colorectal cancer (mCRC) trial. J Clin Oncol 23:278s, 2005 (suppl; abstr 3630)

2. Cunningham $D$, Humblet $Y$, Siena $S$, et al: Cetuximab monotherapy and cetuximab plus irinotecan in irinotecan-refractory metastatic colorectal cancer. N Engl J Med 351:337-345, 2004

3. Folprecht G, Lutz MP, Schoffski $P$, et al: Cetuximab and irinotecan/5-fluorouracil/folinic acid is a safe combination for the first-line treatment of patients with epidermal growth factor receptor expressing metastatic colorectal carcinoma. Ann Oncol 17:450-456, 2006

4. Goldstein NS, Armin M: Epidermal growth factor receptor immunohistochemical reactivity in patients with American Joint Committee on Cancer Stage IV colon adenocarcinoma: Implications for a standardized scoring system. Cancer 92:1331-1346, 2001

5. Saltz LB, Meropol NJ, Loehrer PJ Sr, et al: Phase II trial of cetuximab in patients with refractory colorectal cancer that expresses the epidermal growth factor receptor. J Clin Oncol 22:1201-1208, 2004

6. Goldstein NI, Prewett M, Zuklys $K$, et al: Biological efficacy of a chimeric antibody to the epidermal growth factor receptor in a human tumor xenograft model. Clin Cancer Res 1:1311-1318, 1995

7. Li S, Schmitz KR, Jeffrey PD, et al: Structural basis for inhibition of the epidermal growth factor receptor by cetuximab. Cancer Cell 7:301311, 2005

8. Kang $X$, Patel D, Ng S, et al: High affinity Fc receptor binding and potent induction of antibodydependent cellular cytotoxicity (ADCC) in vitro by anti-epidermal growth factor receptor antibody cetuximab. J Clin Oncol 25:128s, 2007 (suppl; abstr 3041)

9. Kurai J, Chikumi $H$, Hashimoto $K$, et al: Antibody-dependent cellular cytotoxicity mediated by cetuximab against lung cancer cell lines. Clin Cancer Res 13:1552-1561, 2007
10. Bibeau F, Crapez E, Di Fiore F, et al: Association of Fc-gamma Rlla and Fc-gamma Rllla polymorphisms with clinical outcome in metastatic colorectal cancer patients (mCRC) treated with cetuximab and irinotecan. J Clin Oncol 26:579s, 2008 (suppl; abstr 11004)

11. Jonker DJ, O'Callaghan CJ, Karapetis CS, et al: Cetuximab for the treatment of colorectal cancer. N Engl J Med 357:2040-2048, 2007

12. Scheithauer W, Sobrero A, Lenz H, et al: Cetuximab plus irinotecan in patients with metastatic colorectal cancer $(\mathrm{mCRC})$ failing prior oxaliplatin-based therapy: The EPIC trial. Presented at 14th European Cancer Conference, September 23-27, 2007, BarceIona, Spain (abstr 3.003)

13. Peeters M, Raoul J-L, van Laethem J-L, et al: Cetuximab in combination with irinotecan/5fluorouracil (FU)/folinic acid (FA) (FOLFIRI) in the first-line treatment of metastatic colorectal cancer (mCRC). Presented at 13th European Cancer Conference, October 30-November 3, 2005, Paris, France (abstr 664)

14. Van Cutsem E, Bodoky G, Kyung Roh J, et al: CRYSTAL, a randomized phase III trial of cetuximab plus FOLFIRI vs FOLFIRI in first-line metastatic colorectal cancer (mCRC). Presented at 14th European Cancer Conference, September 23-27, 2007, Barcelona, Spain (abstr 3.001)

15. Arnold D, Hoehler T, Dittrich $C$, et al: Cetuximab in combination with weekly 5 -fluorouracil/folinic acid and oxaliplatin (FUFOX) in untreated patients with advanced colorectal cancer: A phase Ib/ll study of the AIO GI group. Ann Oncol 19:14421449, 2008

16. Tabernero J, Van Cutsem E, Díaz-Rubio E, et al: Phase II trial of cetuximab in combination with fluorouracil, leucovorin and oxaliplatin in the first-line treatment of metastatic colorectal cancer. J Clin Oncol 25:5225-5232, 2007

17. Hamilton SR: Targeted therapy of cancer: New roles for pathologists in colorectal cancer. Mod Pathol 21:S23-S30, 2008 (suppl 2)

18. Amado RG, Wolf $M$, Peeters $M$, et al: Wildtype KRAS is required for panitumumab efficacy in patients with metastatic colorectal cancer. J Clin Oncol 26:1626-1634, 2008

19. De Roock W, Piessevaux H, De Schutter J, et al: KRAS wild-type state predicts survival and is associated to early radiological response in meta- static colorectal cancer treated with cetuximab. Ann Oncol 19:508-515, 2008

20. Di Fiore F, Blanchard F, Charbonnier F, et al: Clinical relevance of KRAS mutation detection in metastatic colorectal cancer treated by cetuximab plus chemotherapy. Br J Cancer 96:1166-1169, 2007

21. Lièvre $A$, Bachet $J B$, Boige $V$, et al: KRAS mutations as an independent prognostic factor in patients with advanced colorectal cancer treated with cetuximab. J Clin Oncol 26:374-379, 2008

22. Lièvre $A$, Bachet JB, Le Corre $D$, et al: KRAS mutation status is predictive of response to cetuximab therapy in colorectal cancer. Cancer Res 66: 3992-3995, 2006

23. Van Cutsem E, Lang I, D'haens G, et al: KRAS status and efficacy in the first-line treatment of patients with metastatic colorectal cancer (mCRC) treated with FOLFIRI with or without cetuximab: The CRYSTAL experience. J Clin Oncol 26:5s, 2008 (suppl; abstr 2)

24. Karapetis CS, Khambata-Ford S, Jonker DJ, et al: K-ras mutations and benefit from cetuximab in advanced colorectal cancer. N Engl J Med 359:17571765, 2008

25. Mantel N, Haenszel W: Statistical aspects of the analysis of data from retrospective studies of disease. J Natl Cancer Inst 22:719-748, 1959

26. Mantel N: Chi-square tests with one degree of freedom: Extensions of the Mantel- Haenszel procedure. J Am Stat Assoc 58:690-700, 1963

27. Kaplan EL, Meier P: Nonparametric estimation from incomplete observations J Am Stat Assoc 53:457-481, 1958

28. Chen CY, Shiesh SC, Wu SJ: Rapid detection of K-ras mutations in bile by peptide nucleic acid-mediated PCR clamping and melting curve analysis: Comparison with restriction fragment length polymorphism analysis. Clin Chem 50:481489, 2004

29. Hummelshoj L, Ryder LP, Madsen HO, et al: Locked nucleic acid inhibits amplification of contaminating DNA in real-time PCR. Biotechniques 38:605610, 2005

30. Prewett $M$, Bassi $R$, Carrick $F$, et al: Cetuximab (Erbitux) enhances clinical efficacy of oxaliplatin in human colon carcinoma xenografts and 
reverses oxaliplatin resistance. Presented at AACR$\mathrm{NCl}$-EORTC International Conference, November 14-18, 2005 Philadelphia, PA (abstr B68)

31. Punt CJ, Tol J, Rodenburg CJ, et al: Randomized phase III study of capecitabine, oxaliplatin, and bevacizumab with or without cetuximab in advanced colorectal cancer (ACC), the CAIRO2 study of the Dutch Colorectal Cancer Group (DCCG). J Clin Oncol 26:180s, 2008 (suppl; abst LBA4011)

32. Tabernero J, Cervantes A, Ciardiello F, et al Correlation of efficacy to KRAS status (wt vs. mut) in patients (pts) with metastatic colorectal cancer (mCRC), treated with weekly $(\mathrm{q} 1 \mathrm{w})$ and $q 2 \mathrm{w}$ schedules of cetuximab combined with FOLFIRI. Presented at ASCO Gastrointestinal Cancers Symposium, January 25-27, 2008, Orlando, FL (abstr 435)

\section{Acknowledgment}

We thank the patients enrolled, the medical staff who contributed to their care, and the study team at Merck KGaA, particularly Esther Prang, Barbara Kraus, and Ilhan Celik. We also like thank the investigators at the 87 centers who recruited patients for EGFR expression screening (detailed in the Appendix). 